\title{
Sterile effect in foam concrete
}

\author{
Pavel Gorbach ${ }^{1, *}$, Sergey Shcherbin ${ }^{1}$, and Andrei Savenkov ${ }^{1}$ \\ ${ }^{1}$ Angarsk State Technical University, 665835, Tchaikovsky str., 60, Angarsk, Russia
}

\begin{abstract}
The paper considers the effect of a foaming agent and the steric effect initiated by it on the strength of a cement matrix and foam concrete under the classical technology of producing heat insulating cellular concrete of non-autoclaved hardening.
\end{abstract}

\section{Introduction}

Monolithic non-autoclave foam concrete at present, despite the crisis, is widely used, but it is, in terms of production scale, inferior to the autoclave so far. To increase the volume of production of non-autoclave foam concrete, it is necessary to increase its consumer properties, the main of which is the compressive strength.

The strength of a foam concrete product primarily depends on the strength of its constituent matrix. The matrix is a cement stone without aggregate or with a minimum amount. As the basis of a foam body, it is a material of interporal partitions in a slightly modified form. When surface active substances (surfactants) are added to the cement solution, the surface tension coefficient decreases, and this makes it possible to form a porous structure of cellular concrete. However, the time for seizing the foam concrete is and the growth of its strength are slowing down [1]. In general, the delay is due to the fact that the surfactants create an adsorption layer on the surface of the solvate shells of the cement colloid, initiating the steric repulsion effect (Figure 1).

The concept of a steric effect was first introduced in the middle of the 19th century by Mr. Kermann, who explained the chemical inertness of the substituted quinones with the steric effect of ortho-substituents. Until the 30s of the 20th century, the concept of the steric effect was widely used to explain chemical phenomena by Hoffmann, Menshutkin, Hollemann, Conant, and many other authors [2].

At present, the steric effect is understood to mean a steric obstacle to the course of the reaction provided by a substituent, as a result of a change in the valence angles and the coordination number of the reaction center during the reaction or activation. The concept of shielding the reaction center by a substitute is widely used. In this case, the possibility of spatial convergence of molecules is considered as the most important factor determining the activation energy. For processes occurring in solutions, according to modern concepts, the steric effect (in many cases) reduces to the obstacles that the substituent, for example, surfactants, creates for the solvation of the reaction centers, which are the grains of the binder. Steric effects of substituents can also be manifested indirectly, by inhibiting the formation of new structures [3].

*Corresponding author:pgs@angtu.ru 


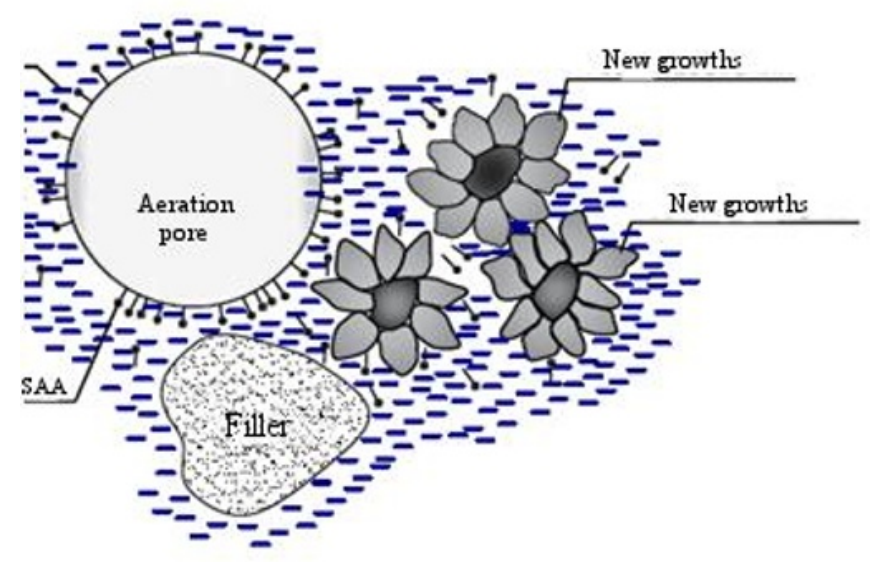

Fig.1. Scheme of manifestation of steric effect.

The presence of this effect is more convenient to experimentally reveal on the matrix, because the influence of the formed large-porous structure is excluded in this case.

Increasing mobility, reducing the water requirement of a concrete mix, or reducing the consumption of cement can be achieved using plasticizing additives. It can be assumed that the foaming agent, as the main component of the foam that forms the structure of the foam concrete at the initial stage, has a significant effect on the characteristics of the cement slurry and the rheological properties of the foam cement mixture [4, 5]. Such mixtures necessarily contain an addition of a foaming agent, the effect of which on the hardening binder is not fully understood. This makes it necessary to study the interaction processes of various foaming agents with cement. The purpose of this work is to study the effect of the steric effect on the structural and mechanical properties of the foam cement mixture and the strength of the foam-concrete monolithic massif.

\section{Materials and methods}

We used the following in our research: (a) the cement PC-500-D0 being produced by the Angarskcement OJSC; (b) synthetic blowing agents, namely Penta Pav 430A and Maxpen; (c) protein foaming agents, Ompor and Bioph; hyperplasticizers Glenium Sky 591, MC Power Flow-3100, and Relamix M.

Determination of the matrix strength was carried out in accordance with GOST 310.481 "Cements: Methods for Determining the Ultimate Strength in Bending and Compression". For manual production of three beam samples, the required amount of binder, as well as surfactants, to which the foaming agents and plasticizers belong, are weighed, and batching is carried out. The resulting homogeneous mass is poured into a metal three-cavity mold being lubricated with oil. After 28 days of normal hardening, the halves of the beams were tested for compression. The tests consist in determining the minimum compressive loads, which destroy half of the standard beams sample of 40x40x160 mm. The compressive strength was determined by testing six halves of beams on a hydraulic press IP-1000. To transfer the load to the beams' halves, flat steel polished plates of $40 \times 62.5 \mathrm{~mm}$ are used. Each half of the beam is placed between the two plates in such a way that the side coincide with the working surfaces, and the abutments of the plates fit snugly against the sample's end smooth wall. Tests on bending were not carried out, since they were not included as part of our research objectives. 


\section{Results}

The nonlinear character of the strength versus porosity dependence, which was expressed in $[6,7,8,9,10]$, was confirmed by the experiments. The graph ( Fig. 2) shows the strength of foam concrete of thermal insulation grades based on the average density of $R b$ on the ratio of the density of foam concrete $\rho_{b}$ and matrix $\rho_{\mathrm{m}}$. With exponential approximation with the reliability $\mathrm{R}^{2}=0.99$ in the range of $\rho_{\mathrm{b}} / \rho_{\mathrm{m}} \leq 0.5$, it corresponds to the following analytical expression:

$$
R b=0,2 \operatorname{Exp}\left(7,2 \rho_{\mathrm{b}} / \rho_{\mathrm{m}}\right)
$$

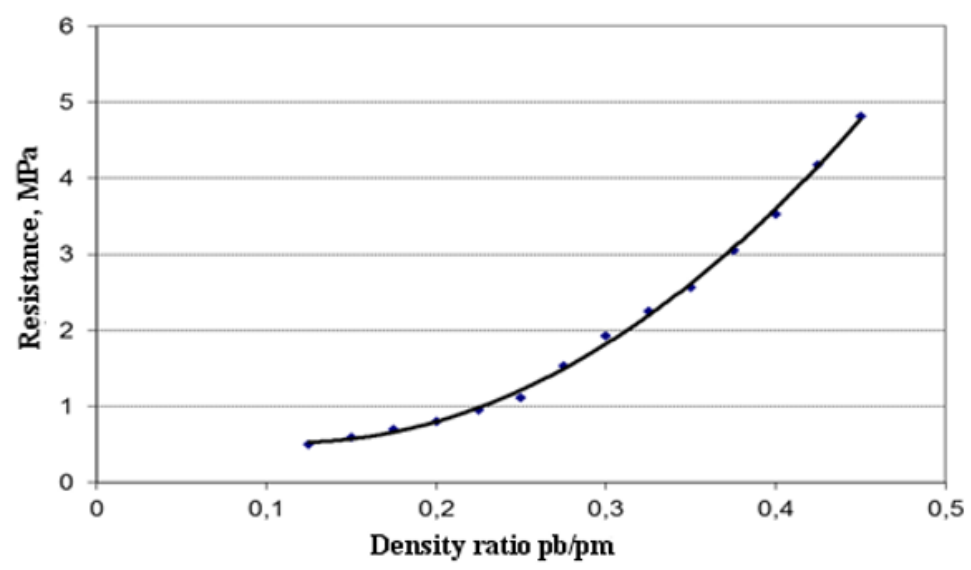

Fig. 2. Dependence of the strength of foam concrete on the ratio of the densities of the foamed cement stone $\rho_{b}$ and the matrix $\rho_{\mathrm{m}}$.

Figure 3 shows the results obtained in determining the strength in samples containing foaming agents of various types. All samples showed a tendency to decrease in strength and density with increasing concentration of foaming agent. The smallest loss of strength was manifested in samples containing the Ompor foaming agent.

In the matrix, the steric effect is expressed in a decrease in density and strength $[3,12]$. Difficulty in the growth of neoplasms leads to loosening of the cement stone's structure, as well as to the formation of interconnected micropores, decreasing in density and, accordingly, in strength (Fig. 3).

When two surfactants are simultaneously present in the cement colloid, namely a foaming agent and a hyperplasticizer, the latter, as the more active surfactant, partially displaces the foaming agent from the solid-liquid interface. This allows, first, to reduce the water requirement of the mixture, and secondly, to reduce the negative effect of the steric effect $[3,4]$.

Both these phenomena have a positive effect on the strength index, which is clearly illustrated in Figure 4. 

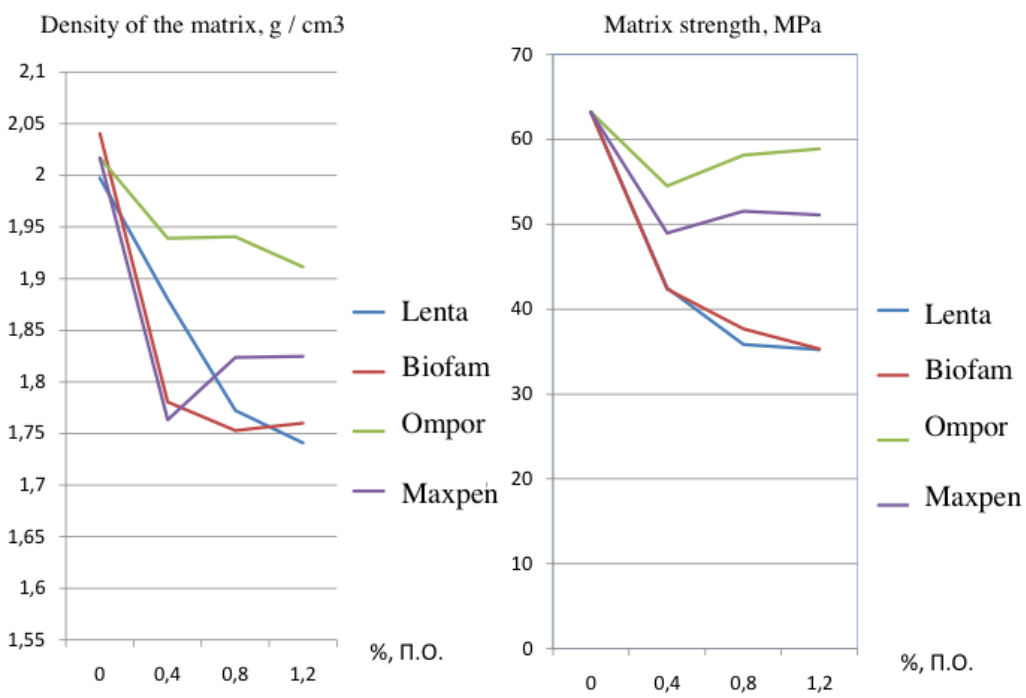

Fig. 3. Dependences of the matrix strength and density on the concentration of surfactants.

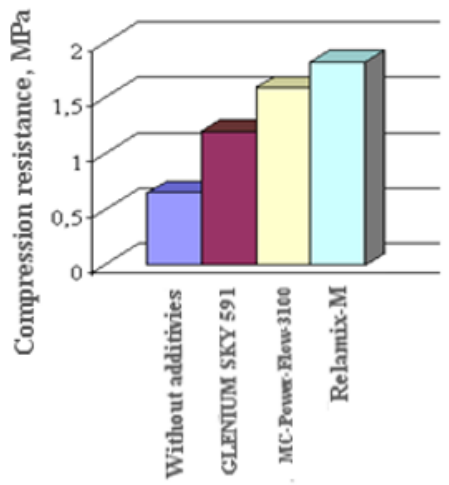

Fig. 4. Comparison of compressive strength of foam concrete samples of density D500 without additives and with additives of plasticizers.

\section{Discussion}

The sterile effect partially inhibits the course of the hydration reaction. Thus, the presence of surface-active substances close to the reacting atoms may prevent these atoms from converging and slow down the reaction, or completely make it impossible. Frothers cause a slowing down of the interaction of $\mathrm{C}_{3} \mathrm{~A}$ with water and change the rate of forming hexagonal hydrate phases. Moreover, large dosages of additives exert a more significant effect on the kinetics of crystallization of hydrate neoplasms and, to a lesser extent, affect the phase composition. In solutions of foaming agents, the process of interaction of $\mathrm{C}_{3} \mathrm{~A}$ with water does not take place completely, and the original alite remains in the system 28 days after hydration.

Before the solidification, the foam cement mixture is a heterogeneous free-disperse system, including solid, liquid, and gaseous phases, in which the dispersed phase is mobile. 
Two dispersed mobile phases can be distinguished: the dispersed gas phase in the dispersion medium and the dispersed gel phase in the aqueous solution in the form of a dispersion medium. Particles in this system are closely approximated; therefore, this system can be conditionally referred to a free-dispersed concentrated system. It is possible that in the foam concrete mixes at the level of thin films of the interporal partitions, microscopic stratifications occur, forming zones in the solid-liquid system with different water contents, in which the separation of the solid-phase particles in size takes place. Over time, it passes into a cohesive dispersed system with a solid dispersed medium, i.e. a cement stone. This cement stone, formed during hardening of general cement, is characterized by a complex stressful state, which can lead to the emergence and development of microcracks.

The effect of hyperplasticizers is based on the joint manifestation of the electrostatic and steric effects that arise with the side hydrophobic polyester chains of the polycarboxylate ester molecule [11]. Due to this, their water-reducing effect reaches more than $50 \%$. Thus, the mechanism of action of hyperplasticizers on cement systems is provided due to the predominant steric effect, which is also characteristic of the presence of surface active substances (Figure 5).

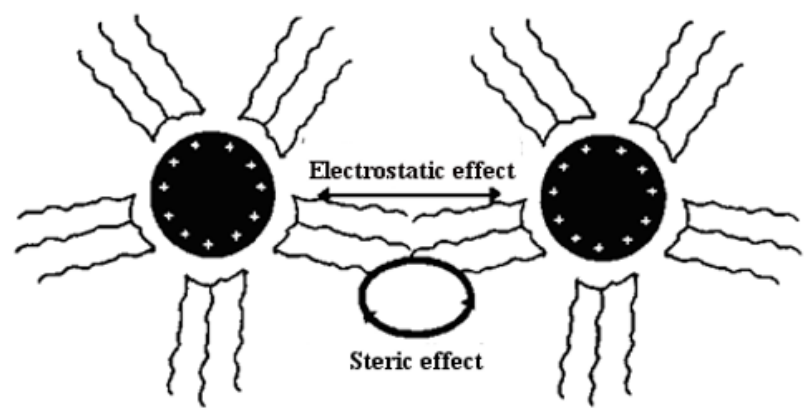

Fig. 5. Scheme of actimg hyperplasticizers in cement mortar.

With this effect of hyperplasticizers, there are a great diluting ability with low dosages of the active substance, reduced sensitivity to the quality of cement, uniformity of concrete and mortar mixtures, and high performance characteristics of the finished product.

Plasticizing additives, adsorbed at the water-solid interface, affect the surface tension of the liquid phase insignificantly. Hyperplasticizers have a dispersant effect, i.e. they increase the amount of particles in the solution and reduce their size, increasing the sedimentation resistance of the cement system $[12,13,14]$.

\section{Conclusion}

There is a nonlinear relationship between the porous structure and the strength of the foamed body (Figure 2). This is due to the joint action of several processes and phenomena. The low strength of the expanded cement stone in comparison with the strength of the matrix and the nonlinearity of the functional dependence of the strength on the average density are due to two groups of factors: the steric repulsion effect in the matrix solution and the large cellular structure, which, for its formation, requires an increased mobility of the mixture.

Therefore, when laying a foam concrete mixture, the steric effect caused by the plasticizer and foaming agent plays a positive role, since it increases the mobility of the solution. But when grasping and typing strength, their action varies considerably. The difference lies in the fact that the plasticizers act temporarily in the solution and then enter 
into a physico-chemical interaction with the hydration products; and the foaming agents remain inert with respect to the solution and make it difficult to grasp and increase the strength of the cement stone.

The established mechanism of the positive effect of the investigated hyperplasticizers on the strength of the material can be used in the production of monolithic commercial foam concrete of heat-insulating grades by density.

\section{References}

1. A. A. Baranova, A. I. Savenkov, Izvestiya Sochi State University, 3(31), 10-14 (2014)

2. L. D. Shakhova, V. V. Balyasnikov, T. I. Chernaya and others, II Intern. Meeting on chemistry and technology of cement (Publishing House of the Central Information Office "Education Information", St. Petersburg, 2000)

3. L. D. Shakhova, Technologies of foam concrete. Theory and practice (ACB, Moscow, 2010)

4. P. S. Gorbach, S. A. Shcherbin, Bulletin of TGASU, 4, 191-199 (2012)

5. V. I. Peskov, K. A. Otsokov, Construction and Building Materials, 3 (2004)

6. D. M. Roy, G. R. Gauda, J. Am. Ceram. Soc, 56 (1973)

7. E. P. Kirsleigh, P. J. Wainwright, J. Cem. Concr. Res., 32, 233-239 (2002)

8. EK K. Nambiar, K. Ramamurthy, J. Mater. Struct., 41, 247-254 (2008)

9. H. Chen, W. Wu, J. Zhou, Construction and Building Materials, 40, 869-874 (2013)

10. A. N. Harhardin, News of higher educational institutions. Construction, 5, 18-25 (2005)

11. Sh. M. Rakhimbaev, D. V. Tverdokhlebov, V. N. Tarasenko. Rheological properties of foam concrete, prepared using foaming agents "Penostrom" and "Neopor". Porobeton 2005. BSTU named after V.G. Shukhov, 143, (2005)

12. M. M. Sychev, The sixth international congress on chemistry of cement (Stroyizdat, Moscow, 1976)

13. V. S. Rachmadran, R. F. Feldman, Additives in concrete (Stroiizdat, Moscow, 1988)

14. R. A. Gadzhily, A. P. Merkin, Surface-active substances in construction (Azerbaijan State Publishing House, Baku, 1981)

15. F. L. Glekel, R. Z. Kopp, K. S. Akhmedov, Regulation of hydration structure formation by surface-active substances (FAN Publishing House of UzSSR, Tashkent, 1986) 